Acta Crystallographica Section E

Structure Reports

Online

ISSN 1600-5368

\section{Tetra- $\mu_{2}$-acetato-diaquabis $\left(\mu_{2}-2-\right.$}

\{[1,3-dihydroxy-2-(oxidomethyl)propan-2-yl]iminomethyl\}phenolato)trimanganese(II,III) acetonitrile disolvate dihydrate

\section{Yuhua Guo, Jianping Huang, Yong Huang, Junyue Wang and Youzhu Yu*}

Department of Chemistry and Environmental Engineering, Anyang Institute of Technology, Henan 455000, People's Republic of China

Correspondence e-mail: 119yyz@163.com

Received 26 June 2011; accepted 12 July 2011

Key indicators: single-crystal X-ray study; $T=293 \mathrm{~K}$; mean $\sigma(\mathrm{C}-\mathrm{C})=0.006 \AA$; $R$ factor $=0.047 ; w R$ factor $=0.133 ;$ data-to-parameter ratio $=18.7$.

In the title complex, $\left[\mathrm{Mn}^{\mathrm{II}} \mathrm{Mn}_{2}{ }_{2}{ }_{2}\left(\mathrm{C}_{11} \mathrm{H}_{13} \mathrm{NO}_{4}\right)_{2}\left(\mathrm{CH}_{3} \mathrm{CO}_{2}\right)_{4}\right.$ $\left.\left(\mathrm{H}_{2} \mathrm{O}\right)_{2}\right] \cdot 2 \mathrm{CH}_{3} \mathrm{CN} \cdot 2 \mathrm{H}_{2} \mathrm{O}$, there are two $\mathrm{Mn}^{\mathrm{III}}$ and one $\mathrm{Mn}^{\mathrm{II}}$ atoms. The $\mathrm{Mn}^{\mathrm{II}}$ atom lies on an inversion center and the $\mathrm{Mn}^{\mathrm{III}}-\mathrm{Mn}^{\mathrm{II}}-\mathrm{Mn}^{\mathrm{III}}$ angle is therefore $180^{\circ}$, as required by crystallographic symmetry. The $\mathrm{Mn}^{\mathrm{III}}$ and $\mathrm{Mn}^{\mathrm{II}}$ atoms are sixcoordinated in a distorted octahedral geometry. In the crystal, complex molecules and solvent molecules are linked into a three-dimensional network by $\mathrm{O}-\mathrm{H} \cdots \mathrm{O}$ and $\mathrm{O}-\mathrm{H} \cdots \mathrm{N}$ hydrogen-bonding interactions.

\section{Related literature}

For the importance of Mn complexes in magnetism and biomimetics, see: Stamatatos \& Christou (2009); Ferreira et al. (2004). For properties and structures of related compounds, see: Kessissoglou et al. (1992); Liu et al. (2010).

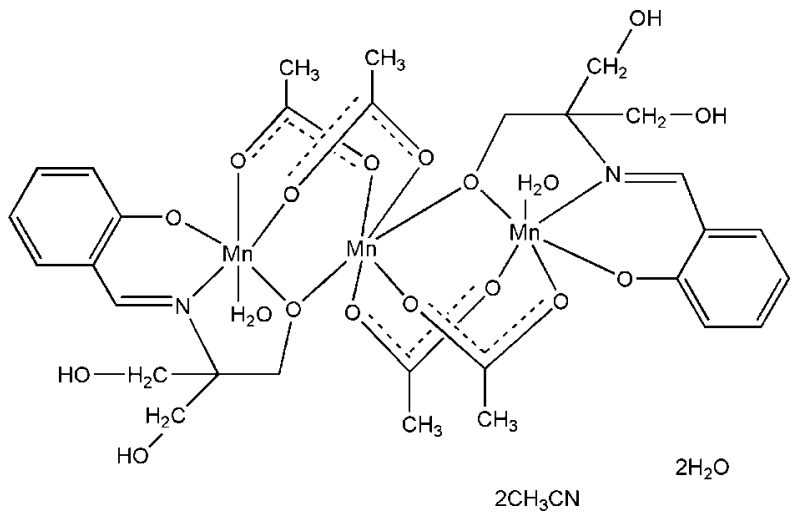

\section{Experimental}

Crystal data

$\left[\mathrm{Mn}_{3}\left(\mathrm{C}_{11} \mathrm{H}_{13} \mathrm{NO}_{4}\right)_{2}\left(\mathrm{C}_{2} \mathrm{H}_{3} \mathrm{O}_{2}\right)_{4}-\right.$

$\left.\left(\mathrm{H}_{2} \mathrm{O}\right)_{2}\right] \cdot 2 \mathrm{C}_{2} \mathrm{H}_{3} \mathrm{~N} \cdot 2 \mathrm{H}_{2} \mathrm{O}$

$M_{r}=1001.62$

Monoclinic, $P 2_{1} / c$

$a=10.6032(5) \AA$

$b=12.2114(6) \AA$

$c=19.1608(9) \AA$

$$
\begin{aligned}
& \beta=118.856(3)^{\circ} \\
& V=2172.89(18) \AA^{3} \\
& Z=2 \\
& \text { Mo } K \alpha \text { radiation } \\
& \mu=0.94 \mathrm{~mm}^{-1} \\
& T=293 \mathrm{~K} \\
& 0.20 \times 0.20 \times 0.20 \mathrm{~mm}
\end{aligned}
$$

\section{Data collection}

Bruker APEXII CCD

diffractometer

53356 measured reflections

5507 independent reflections 3798 reflections with $I>2 \sigma(I)$

$R_{\text {int }}=0.086$

Refinement

$R\left[F^{2}>2 \sigma\left(F^{2}\right)\right]=0.047$

$w R\left(F^{2}\right)=0.133$

\begin{tabular}{|c|c|c|c|c|}
\hline$D-\mathrm{H} \cdots A$ & $D-\mathrm{H}$ & $\mathrm{H} \cdots A$ & $D \cdots A$ & $D-\mathrm{H} \cdots A$ \\
\hline $\mathrm{O} 9-\mathrm{H} 9 A \cdots \mathrm{O} 10^{\mathrm{i}}$ & $0.85(1)$ & 1.97 (1) & $2.806(4)$ & $167(3)$ \\
\hline $\mathrm{O} 9-\mathrm{H} 9 B \cdots \mathrm{O} 8^{\mathrm{ii}}$ & $0.85(1)$ & $2.23(3)$ & $3.008(3)$ & $153(5)$ \\
\hline $\mathrm{O} 9-\mathrm{H} 9 B \cdots \mathrm{O} 1^{\mathrm{ii}}$ & $0.85(1)$ & $2.61(3)$ & $3.322(3)$ & $142(5)$ \\
\hline $\mathrm{O} 10-\mathrm{H} 10 C \cdots \mathrm{O} 5^{\mathrm{iii}}$ & $0.85(1)$ & $2.06(1)$ & $2.907(4)$ & $176(5)$ \\
\hline $\mathrm{O} 10-\mathrm{H} 10 D \cdots \mathrm{N} 2^{\text {iv }}$ & $0.85(1)$ & $2.07(1)$ & $2.914(6)$ & $174(6)$ \\
\hline $\mathrm{O} 2-\mathrm{H} 2 \cdots \mathrm{O} 3^{\mathrm{v}}$ & 0.82 & 2.55 & $3.362(5)$ & 172 \\
\hline $\mathrm{O} 3-\mathrm{H} 3 \cdots \mathrm{O}^{\mathrm{vi}}$ & 0.82 & 2.00 & $2.777(3)$ & 159 \\
\hline
\end{tabular}

$S=1.07$

5507 reflections

294 parameters

7 restraints

Table 1

Hydrogen-bond geometry $\left(\AA{ }^{\circ}\right)$.

Symmetry codes: (i) $x, y+1, z$; (ii) $-x,-y+2,-z+2$; (iii) $-x+1,-y+1,-z+2$; (iv) $x,-y+\frac{3}{2}, z+\frac{1}{2}$; (v) $-x, y-\frac{1}{2},-z+\frac{3}{2}$; (vi) $-x, y+\frac{1}{2},-z+\frac{3}{2}$.

Data collection: APEX2 (Bruker, 1996); cell refinement: SAINT (Bruker, 1996); data reduction: $S A I N T$; program(s) used to solve structure: SHELXS97 (Sheldrick, 2008); program(s) used to refine structure: SHELXL97 (Sheldrick, 2008); molecular graphics: SHELXTL (Sheldrick, 2008); software used to prepare material for publication: SHELXTL.

This work was supported by the Second Young-Aged Backbone Teachers Foundation of Anyang Institute of Technology.

Supplementary data and figures for this paper are available from the IUCr electronic archives (Reference: PV2425).

\title{
References
}

Bruker (1996). APEX2 and SAINT. Bruker AXS Inc., Madison, Wisconsin, USA.

Ferreira, K. N., Iverson, T. M., Maghlaoui, K., Barber, J. \& Iwata, S. (2004). Science, 303, 1831-1838.

Kessissoglou, D. P., Kirk, M. L., Lah, M. S., Li, X., Raptopoulou, C., Hatfield, W. E. \& Pecoraro, V. L. (1992). Inorg. Chem. 31, 5424-5432.

Liu, D., Zhou, Q., Chen, Y., Yang, F., Yu, Y., Shi, Z. \& Feng, S. (2010). Dalton Trans. 39, 5504-5508.

Sheldrick, G. M. (2008). Acta Cryst. A64, 112-122.

Stamatatos, T. C. \& Christou, G. (2009). Inorg. Chem. pp. 3308-3322. 


\section{supporting information}

Acta Cryst. (2011). E67, m1098 [doi:10.1107/S1600536811027899]

\section{Tetra- $\mu_{2}$-acetato-diaquabis $\left(\mu_{2}-2-\{[1,3-d i h y d r o x y-2-(o x i d o m e t h y l) p r o p a n-2-\right.$ yl]iminomethyl\}phenolato)trimanganese(II,III) acetonitrile disolvate dihydrate}

\section{Yuhua Guo, Jianping Huang, Yong Huang, Junyue Wang and Youzhu Yu}

\section{S1. Comment}

The fascination of inorganic chemists with Mn coordination chemistry over the last two decades or so has been primarily driven by the relevance of Mn-complexes to magnetic and biomimetic fields (Stamatatos \& Christou, 2009; Ferreira et al.2004). As a contribution to these fields, we report here the synthesis and crystal structure of the title compound.

In the title complex (Fig. 1), the $\mathrm{Mn}^{\mathrm{III}}$ and $\mathrm{Mn}^{\mathrm{III}}$ atoms are six-coordinated in a distorted octahedral geometry and the two $\mathrm{Mn}^{\mathrm{III}}$ are in the same coordination environment. The $\mathrm{Mn}(\mathrm{II})$ lies on an inversion center, therefore, the angle $\mathrm{Mn}(\mathrm{III})$ $\mathrm{Mn}(\mathrm{II})-\mathrm{Mn}(\mathrm{III})$ is $180^{\circ}$ as required by crystallographic symmetry. The bond lengths and bond angles in the title complex are comparable with those observed in the related complexes (Kessissoglou et al., 1992). In the crystal structure, the complex molecules and the solvent molecules are linked through intermolecular $\mathrm{O}-\mathrm{H} \cdots \mathrm{O}$ and $\mathrm{O}-\mathrm{H} \cdots \mathrm{N}$ hydrogen bonds (Table 1) into a three-dimensional network.

\section{S2. Experimental}

To a stirred acetonitrile $(20 \mathrm{ml})$ solution of $\mathrm{H}_{2} \mathrm{SALATHM}(1 \mathrm{mmol}, 225 \mathrm{mg})$ was added $\mathrm{Mn}(\mathrm{OAc})_{2} \cdot 4 \mathrm{H}_{2} \mathrm{O}(1 \mathrm{mmol}, 245$ $\mathrm{mg}$ ). The resulting dark-red solution was stirred for $1 \mathrm{~h}$ and the filtrate was allowed to stand at room temperature for about three days, whereupon dark block crystal suitable for X-ray diffraction analysis was obtained.

\section{S3. Refinement}

$\mathrm{H}$ atoms were placed at calculated positions with $\mathrm{O}-\mathrm{H}=0.82 \AA$ (hydroxyl), and $\mathrm{C}-\mathrm{H}=0.93 \AA$ (aryl), 0.97 (methylene) and $0.96 \AA$ (methyl) and were refined in the riding-model approximation with $U_{\text {iso }}=1.2-1.5$ times $U_{\text {eq }}$ of the parent atoms. The $\mathrm{H}$-atoms of water of solvation were located from a difference map and were included at distances 0.85 (1) using DFIX commands and were allowed $U_{\text {iso }}=1.5$ times $U_{\mathrm{eq}}(\mathrm{O})$. 
N2
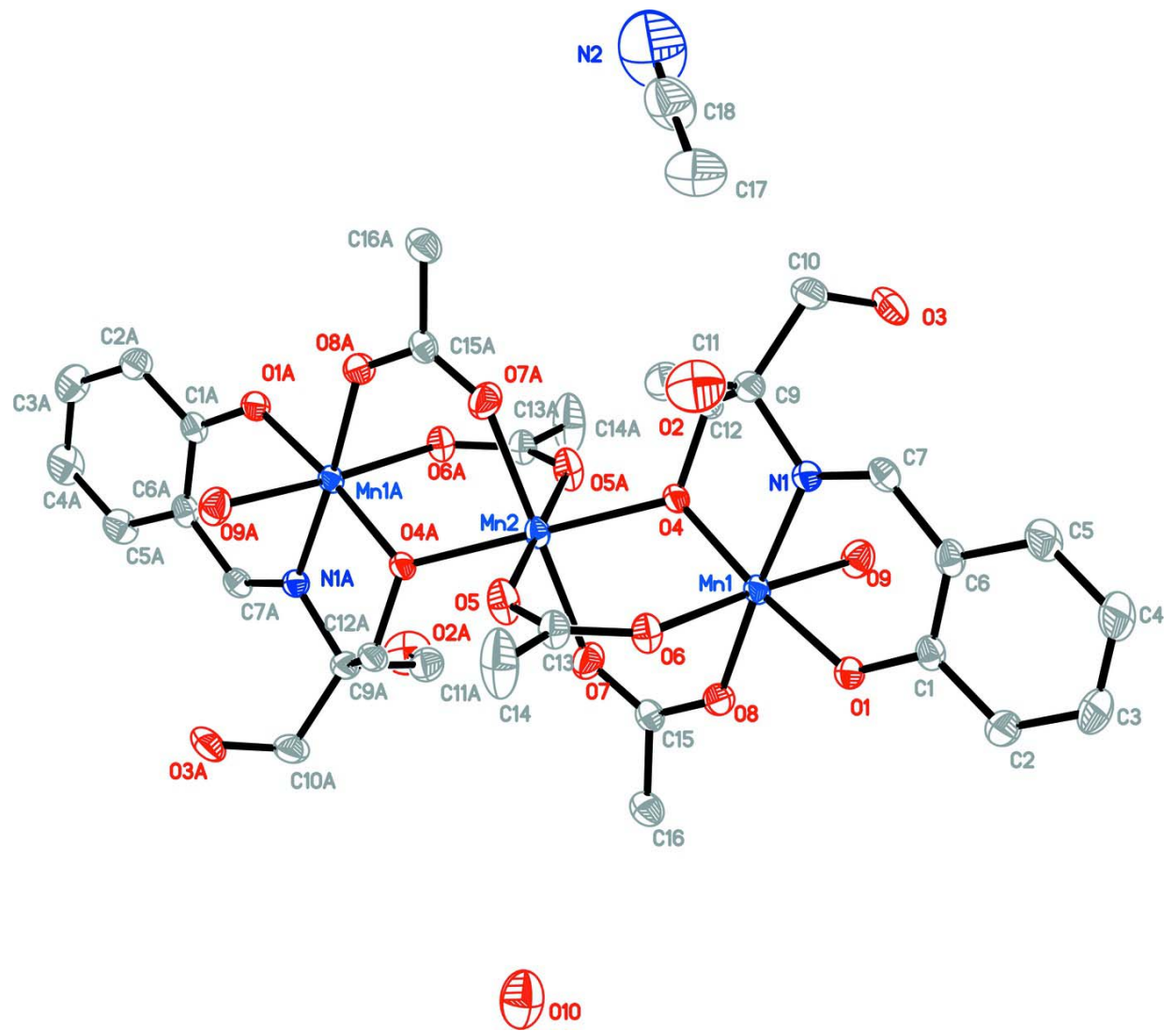

Figure 1

The molecular structure of the title compound, with atom labels and 30\% probability displacement ellipsoids.

Tetra- $\mu_{2}$-acetato-diaquabis $\left(\mu_{2}\right.$-2-\{[1,3-dihydroxy-2- (oxidomethyl)propan-2-

yl] iminomethyl\}phenolato)trimanganese(II,III) acetonitrile disolvate dihydrate

Crystal data

$\left[\mathrm{Mn}_{3}\left(\mathrm{C}_{11} \mathrm{H}_{13} \mathrm{NO}_{4}\right)_{2}\left(\mathrm{C}_{2} \mathrm{H}_{3} \mathrm{O}_{2}\right)_{4}\left(\mathrm{H}_{2} \mathrm{O}\right)_{2}\right] \cdot 2 \mathrm{C}_{2} \mathrm{H}_{3} \mathrm{~N} \cdot 2 \mathrm{H}_{2} \mathrm{O}$

$M_{r}=1001.62$

Monoclinic, $P 2{ }_{1} / c$

Hall symbol: -P $2 \mathrm{ybc}$

$a=10.6032(5) \AA$

$b=12.2114(6) \AA$

$c=19.1608(9) \AA$

$\beta=118.856(3)^{\circ}$

$V=2172.89(18) \AA^{3}$

$Z=2$

$$
F(000)=1038
$$

$D_{\mathrm{x}}=1.531 \mathrm{Mg} \mathrm{m}^{-3}$

Mo $K \alpha$ radiation, $\lambda=0.71073 \AA$

Cell parameters from 9948 reflections

$\theta=2.4-28.4^{\circ}$

$\mu=0.94 \mathrm{~mm}^{-1}$

$T=293 \mathrm{~K}$

Block, black

$0.20 \times 0.20 \times 0.20 \mathrm{~mm}$

\section{Data collection}

\section{Bruker APEXII CCD}

diffractometer

Radiation source: fine-focus sealed tube

Graphite monochromator

$\varphi$ and $\omega$ scans

53356 measured reflections

5507 independent reflections

3798 reflections with $I>2 \sigma(I)$

$R_{\text {int }}=0.086$

$\theta_{\max }=28.6^{\circ}, \theta_{\min }=2.1^{\circ}$

$h=-14 \rightarrow 14$

$k=-16 \rightarrow 16$

$l=-25 \rightarrow 25$ 


\section{Refinement}

Refinement on $F^{2}$

Least-squares matrix: full

$R\left[F^{2}>2 \sigma\left(F^{2}\right)\right]=0.047$

$w R\left(F^{2}\right)=0.133$

$S=1.07$

5507 reflections

294 parameters

7 restraints

Primary atom site location: structure-invariant direct methods
Secondary atom site location: difference Fourier map

Hydrogen site location: inferred from neighbouring sites

$\mathrm{H}$ atoms treated by a mixture of independent and constrained refinement

$w=1 /\left[\sigma^{2}\left(F_{\mathrm{o}}^{2}\right)+(0.0525 P)^{2}+2.7933 P\right]$ where $P=\left(F_{\mathrm{o}}^{2}+2 F_{\mathrm{c}}^{2}\right) / 3$

$(\Delta / \sigma)_{\max }=0.024$

$\Delta \rho_{\max }=0.72 \mathrm{e} \AA^{-3}$

$\Delta \rho_{\min }=-0.61$ e $\AA^{-3}$

Fractional atomic coordinates and isotropic or equivalent isotropic displacement parameters $\left(\AA^{2}\right)$

\begin{tabular}{|c|c|c|c|c|}
\hline & $x$ & $y$ & $z$ & $U_{\text {iso }} * / U_{\text {eq }}$ \\
\hline $\mathrm{C} 1$ & $-0.1569(3)$ & $0.8511(2)$ & $0.84008(18)$ & $0.0317(6)$ \\
\hline $\mathrm{C} 2$ & $-0.2687(4)$ & $0.8090(3)$ & $0.8512(2)$ & $0.0419(8)$ \\
\hline $\mathrm{H} 2 \mathrm{~A}$ & -0.2506 & 0.7888 & 0.9020 & $0.050^{*}$ \\
\hline $\mathrm{C} 3$ & $-0.4053(4)$ & $0.7971(3)$ & $0.7877(2)$ & $0.0548(10)$ \\
\hline $\mathrm{H} 3 \mathrm{~A}$ & -0.4788 & 0.7710 & 0.7966 & $0.066^{*}$ \\
\hline $\mathrm{C} 4$ & $-0.4352(4)$ & 0.8235 (4) & $0.7105(3)$ & 0.0604 (11) \\
\hline $\mathrm{H} 4$ & -0.5274 & 0.8140 & 0.6680 & $0.072 *$ \\
\hline $\mathrm{C} 5$ & $-0.3275(4)$ & 0.8635 & $0.6980(2)$ & $0.0475(9)$ \\
\hline H5 & -0.3469 & 0.8808 & 0.6465 & $0.057^{*}$ \\
\hline C6 & -0.1874 & 0.8789 (2) & $0.76201(18)$ & $0.0345(7)$ \\
\hline $\mathrm{C} 7$ & $-0.0836(3)$ & $0.9291(2)$ & $0.74451(18)$ & $0.0334(6)$ \\
\hline H7 & -0.1103 & 0.9400 & 0.6911 & $0.040^{*}$ \\
\hline $\mathrm{C} 9$ & $0.1393(3)$ & $1.0157(3)$ & $0.77120(18)$ & $0.0352(7)$ \\
\hline $\mathrm{C} 10$ & 0.0585 (4) & $1.0849(3)$ & $0.6962(2)$ & $0.0464(9)$ \\
\hline $\mathrm{H} 10 \mathrm{~A}$ & 0.1265 & 1.1269 & 0.6868 & $0.056^{*}$ \\
\hline H10B & 0.0049 & 1.0376 & 0.6506 & $0.056^{*}$ \\
\hline C11 & $0.2297(4)$ & 0.9275 (3) & $0.7590(2)$ & $0.0486(9)$ \\
\hline H11A & 0.2880 & 0.9621 & 0.7388 & $0.058^{*}$ \\
\hline H11B & 0.2946 & 0.8953 & 0.8102 & $0.058^{*}$ \\
\hline $\mathrm{C} 12$ & $0.2357(3)$ & $1.0870(3)$ & $0.84214(18)$ & $0.0342(7)$ \\
\hline $\mathrm{H} 12 \mathrm{~A}$ & 0.3199 & 1.1091 & 0.8382 & $0.041^{*}$ \\
\hline H12B & 0.1840 & 1.1525 & 0.8422 & $0.041 *$ \\
\hline C13 & $0.3707(3)$ & $0.7594(2)$ & 0.94069 (19) & $0.0357(7)$ \\
\hline $\mathrm{C} 14$ & $0.3997(5)$ & $0.6436(3)$ & 0.9255 (4) & $0.0821(17)$ \\
\hline H14A & 0.4021 & 0.6404 & 0.8761 & $0.123 *$ \\
\hline H14B & 0.3248 & 0.5965 & 0.9226 & $0.123^{*}$ \\
\hline $\mathrm{H} 14 \mathrm{C}$ & 0.4907 & 0.6200 & 0.9682 & $0.123^{*}$ \\
\hline $\mathrm{C} 15$ & $0.3292(3)$ & $0.9424(2)$ & $1.09183(17)$ & $0.0315(6)$ \\
\hline $\mathrm{C} 16$ & $0.3403(4)$ & 0.9114 (3) & $1.1703(2)$ & $0.0440(8)$ \\
\hline H16A & 0.4374 & 0.9225 & 1.2120 & $0.066^{*}$ \\
\hline H16B & 0.3147 & 0.8358 & 1.1690 & $0.066^{*}$ \\
\hline H16C & 0.2761 & 0.9561 & 1.1801 & $0.066^{*}$ \\
\hline C17 & $0.1100(6)$ & $0.8401(4)$ & $0.5308(3)$ & $0.0706(13)$ \\
\hline
\end{tabular}




$\begin{array}{lllll}\text { H17B } & 0.0142 & 0.8391 & 0.4864 & 0.106^{*} \\ \text { H17A } & 0.1057 & 0.8323 & 0.5794 & 0.106^{*} \\ \text { H17C } & 0.1644 & 0.7806 & 0.5258 & 0.106^{*} \\ \text { C18 } & 0.1775(5) & 0.9407(4) & 0.5317(3) & 0.0742(14) \\ \text { H9A } & 0.082(4) & 1.158(2) & 0.938(3) & 0.111^{*} \\ \text { H9B } & -0.014(5) & 1.099(3) & 0.953(3) & 0.111^{*} \\ \text { H10C } & 0.320(2) & 0.242(4) & 0.984(3) & 0.111^{*} \\ \text { H10D } & 0.235(5) & 0.327(3) & 0.987(3) & 0.111^{*} \\ \text { N1 } & 0.0433(3) & 0.9604(2) & 0.79651(14) & 0.0299(5) \\ \text { N2 } & 0.2282(7) & 1.0199(4) & 0.5303(5) & 0.146(3) \\ \text { O1 } & -0.0288(2) & 0.86304(18) & 0.90298(12) & 0.0356(5) \\ \text { O2 } & 0.1473(4) & 0.8430(3) & 0.7059(2) & 0.0775(9) \\ \text { H2 } & 0.1298 & 0.7957 & 0.7305 & 0.116^{*} \\ \text { O3 } & -0.0374(3) & 1.1568(2) & 0.70565(16) & 0.0569(7) \\ \text { H3 } & -0.0849 & 1.1909 & 0.6642 & 0.085^{*} \\ \text { O4 } & 0.2792(2) & 1.02733(16) & 0.91436(11) & 0.0290(4) \\ \text { O5 } & 0.4759(2) & 0.82092(18) & 0.97622(15) & 0.0466(6) \\ \text { O6 } & 0.2412(2) & 0.78562(16) & 0.91590(13) & 0.0359(5) \\ \text { O7 } & 0.4363(2) & 0.9780(2) & 1.08976(14) & 0.0480(6) \\ \text { O8 } & 0.2062(2) & 0.92758(18) & 1.03081(12) & 0.0358(5) \\ \text { O9 } & 0.0281(3) & 1.10148(19) & 0.92495(16) & 0.0451(6) \\ \text { O10 } & 0.2353(3) & 0.2668(3) & 0.9640(2) & 0.0640(8) \\ \text { Mn1 } & 0.13180(4) & 0.94343(3) & 0.91490(2) & 0.02691(13) \\ \text { Mn2 } & 0.5000 & 1.0000 & 1.0000 & 0.02878(16) \\ & & & & \end{array}$

Atomic displacement parameters $\left(\AA^{2}\right)$

\begin{tabular}{lllllll}
\hline & $U^{11}$ & $U^{22}$ & $U^{33}$ & $U^{12}$ & $U^{13}$ & $U^{23}$ \\
\hline C1 & $0.0261(14)$ & $0.0260(14)$ & $0.0358(16)$ & $-0.0014(11)$ & $0.0091(12)$ & $-0.0011(11)$ \\
C2 & $0.0379(17)$ & $0.0424(18)$ & $0.0430(19)$ & $-0.0061(14)$ & $0.0176(15)$ & $0.0013(14)$ \\
C3 & $0.0326(17)$ & $0.061(2)$ & $0.064(3)$ & $-0.0180(17)$ & $0.0181(17)$ & $-0.0055(19)$ \\
C4 & $0.0317(18)$ & $0.069(3)$ & $0.057(2)$ & $-0.0176(18)$ & $0.0032(17)$ & $-0.003(2)$ \\
C5 & $0.0363(18)$ & $0.050(2)$ & $0.0377(19)$ & $-0.0101(15)$ & $0.0030(14)$ & $-0.0016(15)$ \\
C6 & $0.0258(14)$ & $0.0306(15)$ & $0.0382(17)$ & $-0.0024(12)$ & $0.0085(12)$ & $-0.0031(12)$ \\
C7 & $0.0313(15)$ & $0.0351(16)$ & $0.0260(14)$ & $0.0012(12)$ & $0.0075(12)$ & $0.0006(12)$ \\
C9 & $0.0302(15)$ & $0.0398(16)$ & $0.0328(16)$ & $-0.0026(13)$ & $0.0129(13)$ & $0.0056(13)$ \\
C10 & $0.0397(18)$ & $0.055(2)$ & $0.0352(18)$ & $-0.0057(16)$ & $0.0104(15)$ & $0.0132(15)$ \\
C11 & $0.049(2)$ & $0.059(2)$ & $0.042(2)$ & $0.0034(17)$ & $0.0253(17)$ & $-0.0003(16)$ \\
C12 & $0.0273(14)$ & $0.0342(15)$ & $0.0340(16)$ & $-0.0017(12)$ & $0.0092(12)$ & $0.0082(12)$ \\
C13 & $0.0308(15)$ & $0.0274(14)$ & $0.0443(18)$ & $-0.0002(12)$ & $0.0145(14)$ & $-0.0049(13)$ \\
C14 & $0.045(2)$ & $0.040(2)$ & $0.139(5)$ & $0.0000(17)$ & $0.026(3)$ & $-0.030(3)$ \\
C15 & $0.0321(15)$ & $0.0281(14)$ & $0.0313(15)$ & $0.0043(12)$ & $0.0130(12)$ & $-0.0022(12)$ \\
C16 & $0.0445(19)$ & $0.0471(19)$ & $0.0348(18)$ & $0.0055(15)$ & $0.0148(15)$ & $0.0028(14)$ \\
C17 & $0.102(4)$ & $0.049(2)$ & $0.069(3)$ & $-0.006(2)$ & $0.047(3)$ & $-0.004(2)$ \\
C18 & $0.074(3)$ & $0.047(2)$ & $0.087(3)$ & $0.001(2)$ & $0.027(3)$ & $0.011(2)$ \\
N1 & $0.0262(12)$ & $0.0323(13)$ & $0.0284(12)$ & $-0.0012(10)$ & $0.0110(10)$ & $0.0024(10)$ \\
N2 & $0.124(5)$ & $0.065(3)$ & $0.225(8)$ & $-0.021(3)$ & $0.065(5)$ & $0.031(4)$ \\
O1 & $0.0247(10)$ & $0.0422(12)$ & $0.0327(11)$ & $-0.0046(9)$ & $0.0080(9)$ & $0.0057(9)$
\end{tabular}


supporting information

\begin{tabular}{lllllll} 
O2 & $0.101(3)$ & $0.072(2)$ & $0.068(2)$ & $-0.0063(19)$ & $0.047(2)$ & $-0.0187(17)$ \\
O3 & $0.0442(14)$ & $0.0612(17)$ & $0.0493(15)$ & $0.0126(12)$ & $0.0099(12)$ & $0.0258(13)$ \\
O4 & $0.0218(9)$ & $0.0311(10)$ & $0.0286(10)$ & $0.0003(8)$ & $0.0078(8)$ & $0.0061(8)$ \\
O5 & $0.0310(11)$ & $0.0290(11)$ & $0.0633(16)$ & $-0.0016(9)$ & $0.0096(11)$ & $-0.0065(11)$ \\
O6 & $0.0285(10)$ & $0.0283(10)$ & $0.0440(13)$ & $-0.0011(8)$ & $0.0120(9)$ & $-0.0063(9)$ \\
O7 & $0.0313(12)$ & $0.0740(17)$ & $0.0372(13)$ & $-0.0035(12)$ & $0.0152(10)$ & $-0.0003(12)$ \\
O8 & $0.0306(11)$ & $0.0434(12)$ & $0.0291(11)$ & $-0.0049(9)$ & $0.0109(9)$ & $-0.0004(9)$ \\
O9 & $0.0436(14)$ & $0.0384(13)$ & $0.0596(16)$ & $0.0039(10)$ & $0.0299(12)$ & $-0.0016(11)$ \\
O10 & $0.0495(16)$ & $0.0581(18)$ & $0.081(2)$ & $0.0004(13)$ & $0.0286(16)$ & $-0.0148(15)$ \\
Mn1 & $0.0215(2)$ & $0.0281(2)$ & $0.0258(2)$ & $-0.00170(16)$ & $0.00718(17)$ & $0.00117(17)$ \\
Mn2 & $0.0204(3)$ & $0.0280(3)$ & $0.0314(3)$ & $-0.0005(2)$ & $0.0073(2)$ & $-0.0008(2)$ \\
\hline
\end{tabular}

Geometric parameters $\left(\hat{A},{ }^{\circ}\right)$

\begin{tabular}{|c|c|c|c|}
\hline $\mathrm{C} 1-\mathrm{O} 1$ & $1.319(3)$ & C14-H14B & 0.9600 \\
\hline $\mathrm{C} 1-\mathrm{C} 2$ & $1.400(4)$ & $\mathrm{C} 14-\mathrm{H} 14 \mathrm{C}$ & 0.9600 \\
\hline $\mathrm{C} 1-\mathrm{C} 6$ & $1.411(4)$ & $\mathrm{C} 15-\mathrm{O} 7$ & $1.234(4)$ \\
\hline $\mathrm{C} 2-\mathrm{C} 3$ & $1.378(5)$ & $\mathrm{C} 15-\mathrm{O} 8$ & $1.276(3)$ \\
\hline $\mathrm{C} 2-\mathrm{H} 2 \mathrm{~A}$ & 0.9300 & $\mathrm{C} 15-\mathrm{C} 16$ & $1.498(4)$ \\
\hline $\mathrm{C} 3-\mathrm{C} 4$ & $1.393(6)$ & $\mathrm{C} 16-\mathrm{H} 16 \mathrm{~A}$ & 0.9600 \\
\hline $\mathrm{C} 3-\mathrm{H} 3 \mathrm{~A}$ & 0.9300 & C16-H16B & 0.9600 \\
\hline $\mathrm{C} 4-\mathrm{C} 5$ & $1.365(5)$ & $\mathrm{C} 16-\mathrm{H} 16 \mathrm{C}$ & 0.9600 \\
\hline $\mathrm{C} 4-\mathrm{H} 4$ & 0.9300 & $\mathrm{C} 17-\mathrm{C} 18$ & $1.418(6)$ \\
\hline $\mathrm{C} 5-\mathrm{C} 6$ & $1.410(4)$ & C17-H17B & 0.9600 \\
\hline $\mathrm{C} 5-\mathrm{H} 5$ & 0.9300 & C17-H17A & 0.9600 \\
\hline $\mathrm{C} 6-\mathrm{C} 7$ & $1.434(4)$ & $\mathrm{C} 17-\mathrm{H} 17 \mathrm{C}$ & 0.9600 \\
\hline $\mathrm{C} 7-\mathrm{N} 1$ & $1.287(4)$ & $\mathrm{C} 18-\mathrm{N} 2$ & $1.113(6)$ \\
\hline $\mathrm{C} 7-\mathrm{H} 7$ & 0.9300 & $\mathrm{~N} 1-\mathrm{Mn} 1$ & $2.004(2)$ \\
\hline $\mathrm{C} 9-\mathrm{N} 1$ & $1.484(4)$ & $\mathrm{O} 1-\mathrm{Mn} 1$ & $1.882(2)$ \\
\hline C9- $\mathrm{C} 12$ & $1.520(4)$ & $\mathrm{O} 2-\mathrm{H} 2$ & 0.8200 \\
\hline $\mathrm{C} 9-\mathrm{C} 10$ & $1.525(4)$ & $\mathrm{O} 3-\mathrm{H} 3$ & 0.8200 \\
\hline $\mathrm{C} 9-\mathrm{C} 11$ & $1.533(5)$ & $\mathrm{O} 4-\mathrm{Mn} 1$ & $1.8729(19)$ \\
\hline $\mathrm{C} 10-\mathrm{O} 3$ & $1.420(5)$ & $\mathrm{O} 4-\mathrm{Mn} 2$ & $2.1395(18)$ \\
\hline $\mathrm{C} 10-\mathrm{H} 10 \mathrm{~A}$ & 0.9700 & $\mathrm{O} 5-\mathrm{Mn} 2$ & $2.223(2)$ \\
\hline $\mathrm{C} 10-\mathrm{H} 10 \mathrm{~B}$ & 0.9700 & $\mathrm{O} 6-\mathrm{Mn} 1$ & $2.244(2)$ \\
\hline $\mathrm{C} 11-\mathrm{O} 2$ & $1.416(5)$ & $\mathrm{O} 7-\mathrm{Mn} 2$ & $2.147(2)$ \\
\hline C11-H11A & 0.9700 & $\mathrm{O} 8-\mathrm{Mn} 1$ & $1.975(2)$ \\
\hline $\mathrm{C} 11-\mathrm{H} 11 \mathrm{~B}$ & 0.9700 & O9-Mn1 & $2.275(2)$ \\
\hline $\mathrm{C} 12-\mathrm{O} 4$ & $1.429(3)$ & O9-H9A & $0.851(10)$ \\
\hline $\mathrm{C} 12-\mathrm{H} 12 \mathrm{~A}$ & 0.9700 & O9- & $0.851(10)$ \\
\hline $\mathrm{C} 12-\mathrm{H} 12 \mathrm{~B}$ & 0.9700 & $\mathrm{O} 10-\mathrm{H} 10 \mathrm{C}$ & $0.851(10)$ \\
\hline $\mathrm{C} 13-\mathrm{O} 5$ & $1.241(4)$ & $\mathrm{O} 10-\mathrm{H} 10 \mathrm{D}$ & $0.849(10)$ \\
\hline $\mathrm{C} 13-\mathrm{O} 6$ & $1.258(4)$ & $\mathrm{Mn} 2-\mathrm{O} 4^{\mathrm{i}}$ & $2.1395(18)$ \\
\hline $\mathrm{C} 13-\mathrm{C} 14$ & $1.505(5)$ & $\mathrm{Mn} 2-\mathrm{O} 7^{\mathrm{i}}$ & $2.147(2)$ \\
\hline $\mathrm{C} 14-\mathrm{H} 14 \mathrm{~A}$ & 0.9600 & $\mathrm{Mn} 2-\mathrm{O}^{\mathrm{i}}$ & $2.223(2)$ \\
\hline $\mathrm{O} 1-\mathrm{C} 1-\mathrm{C} 2$ & $118.4(3)$ & $\mathrm{H} 16 \mathrm{~A}-\mathrm{C} 16-\mathrm{H} 16 \mathrm{~B}$ & 109.5 \\
\hline $\mathrm{O} 1-\mathrm{C} 1-\mathrm{C} 6$ & $123.4(3)$ & $\mathrm{C} 15-\mathrm{C} 16-\mathrm{H} 16 \mathrm{C}$ & 109.5 \\
\hline
\end{tabular}




\begin{tabular}{|c|c|c|c|}
\hline $\mathrm{C} 2-\mathrm{C} 1-\mathrm{C} 6$ & $118.2(3)$ & $\mathrm{H} 16 \mathrm{~A}-\mathrm{C} 16-\mathrm{H} 16 \mathrm{C}$ & 109.5 \\
\hline $\mathrm{C} 3-\mathrm{C} 2-\mathrm{C} 1$ & $120.7(3)$ & $\mathrm{H} 16 \mathrm{~B}-\mathrm{C} 16-\mathrm{H} 16 \mathrm{C}$ & 109.5 \\
\hline $\mathrm{C} 3-\mathrm{C} 2-\mathrm{H} 2 \mathrm{~A}$ & 119.7 & $\mathrm{C} 18-\mathrm{C} 17-\mathrm{H} 17 \mathrm{~B}$ & 109.5 \\
\hline $\mathrm{C} 1-\mathrm{C} 2-\mathrm{H} 2 \mathrm{~A}$ & 119.7 & $\mathrm{C} 18-\mathrm{C} 17-\mathrm{H} 17 \mathrm{~A}$ & 109.5 \\
\hline $\mathrm{C} 2-\mathrm{C} 3-\mathrm{C} 4$ & $121.1(3)$ & $\mathrm{H} 17 \mathrm{~B}-\mathrm{C} 17-\mathrm{H} 17 \mathrm{~A}$ & 109.5 \\
\hline $\mathrm{C} 2-\mathrm{C} 3-\mathrm{H} 3 \mathrm{~A}$ & 119.4 & $\mathrm{C} 18-\mathrm{C} 17-\mathrm{H} 17 \mathrm{C}$ & 109.5 \\
\hline $\mathrm{C} 4-\mathrm{C} 3-\mathrm{H} 3 \mathrm{~A}$ & 119.4 & $\mathrm{H} 17 \mathrm{~B}-\mathrm{C} 17-\mathrm{H} 17 \mathrm{C}$ & 109.5 \\
\hline $\mathrm{C} 5-\mathrm{C} 4-\mathrm{C} 3$ & $119.3(3)$ & $\mathrm{H} 17 \mathrm{~A}-\mathrm{C} 17-\mathrm{H} 17 \mathrm{C}$ & 109.5 \\
\hline $\mathrm{C} 5-\mathrm{C} 4-\mathrm{H} 4$ & 120.4 & $\mathrm{~N} 2-\mathrm{C} 18-\mathrm{C} 17$ & $178.1(7)$ \\
\hline $\mathrm{C} 3-\mathrm{C} 4-\mathrm{H} 4$ & 120.4 & $\mathrm{C} 7-\mathrm{N} 1-\mathrm{C} 9$ & $120.5(3)$ \\
\hline $\mathrm{C} 4-\mathrm{C} 5-\mathrm{C} 6$ & $120.9(3)$ & $\mathrm{C} 7-\mathrm{N} 1-\mathrm{Mn} 1$ & $126.1(2)$ \\
\hline $\mathrm{C} 4-\mathrm{C} 5-\mathrm{H} 5$ & 119.6 & $\mathrm{C} 9-\mathrm{N} 1-\mathrm{Mn} 1$ & $113.37(18)$ \\
\hline $\mathrm{C} 6-\mathrm{C} 5-\mathrm{H} 5$ & 119.6 & $\mathrm{C} 1-\mathrm{O} 1-\mathrm{Mn} 1$ & $129.90(19)$ \\
\hline $\mathrm{C} 5-\mathrm{C} 6-\mathrm{C} 1$ & $119.8(3)$ & $\mathrm{C} 11-\mathrm{O} 2-\mathrm{H} 2$ & 109.5 \\
\hline $\mathrm{C} 5-\mathrm{C} 6-\mathrm{C} 7$ & $117.3(3)$ & $\mathrm{C} 10-\mathrm{O} 3-\mathrm{H} 3$ & 109.5 \\
\hline $\mathrm{C} 1-\mathrm{C} 6-\mathrm{C} 7$ & $122.8(3)$ & $\mathrm{C} 12-\mathrm{O} 4-\mathrm{Mn} 1$ & $113.83(16)$ \\
\hline $\mathrm{N} 1-\mathrm{C} 7-\mathrm{C} 6$ & $125.4(3)$ & $\mathrm{C} 12-\mathrm{O} 4-\mathrm{Mn} 2$ & $122.99(17)$ \\
\hline $\mathrm{N} 1-\mathrm{C} 7-\mathrm{H} 7$ & 117.3 & $\mathrm{Mn} 1-\mathrm{O} 4-\mathrm{Mn} 2$ & $121.17(9)$ \\
\hline $\mathrm{C} 6-\mathrm{C} 7-\mathrm{H} 7$ & 117.3 & $\mathrm{C} 13-\mathrm{O} 5-\mathrm{Mn} 2$ & $133.8(2)$ \\
\hline $\mathrm{N} 1-\mathrm{C} 9-\mathrm{C} 12$ & $103.9(2)$ & $\mathrm{C} 13-\mathrm{O} 6-\mathrm{Mn} 1$ & $133.38(19)$ \\
\hline $\mathrm{N} 1-\mathrm{C} 9-\mathrm{C} 10$ & $113.5(3)$ & $\mathrm{C} 15-\mathrm{O} 7-\mathrm{Mn} 2$ & $136.1(2)$ \\
\hline $\mathrm{C} 12-\mathrm{C} 9-\mathrm{C} 10$ & $110.8(3)$ & $\mathrm{C} 15-\mathrm{O} 8-\mathrm{Mn} 1$ & $133.9(2)$ \\
\hline $\mathrm{N} 1-\mathrm{C} 9-\mathrm{C} 11$ & $108.0(3)$ & $\mathrm{Mn} 1-\mathrm{O} 9-\mathrm{H} 9 \mathrm{~A}$ & $116(2)$ \\
\hline $\mathrm{C} 12-\mathrm{C} 9-\mathrm{C} 11$ & $109.8(3)$ & Mn1-O9-H9B & $116(3)$ \\
\hline $\mathrm{C} 10-\mathrm{C} 9-\mathrm{C} 11$ & $110.7(3)$ & $\mathrm{H} 9 \mathrm{~A}-\mathrm{O} 9-\mathrm{H} 9 \mathrm{~B}$ & $109(2)$ \\
\hline $\mathrm{O} 3-\mathrm{C} 10-\mathrm{C} 9$ & $109.5(3)$ & $\mathrm{H} 10 \mathrm{C}-\mathrm{O} 10-\mathrm{H} 10 \mathrm{D}$ & $110(3)$ \\
\hline $\mathrm{O} 3-\mathrm{C} 10-\mathrm{H} 10 \mathrm{~A}$ & 109.8 & $\mathrm{O} 4-\mathrm{Mn} 1-\mathrm{O} 1$ & $173.45(9)$ \\
\hline $\mathrm{C} 9-\mathrm{C} 10-\mathrm{H} 10 \mathrm{~A}$ & 109.8 & $\mathrm{O} 4-\mathrm{Mn} 1-\mathrm{O} 8$ & $100.27(9)$ \\
\hline $\mathrm{O} 3-\mathrm{C} 10-\mathrm{H} 10 \mathrm{~B}$ & 109.8 & $\mathrm{O} 1-\mathrm{Mn} 1-\mathrm{O} 8$ & $86.11(9)$ \\
\hline $\mathrm{C} 9-\mathrm{C} 10-\mathrm{H} 10 \mathrm{~B}$ & 109.8 & $\mathrm{O} 4-\mathrm{Mn} 1-\mathrm{N} 1$ & $82.72(9)$ \\
\hline $\mathrm{H} 10 \mathrm{~A}-\mathrm{C} 10-\mathrm{H} 10 \mathrm{~B}$ & 108.2 & $\mathrm{O} 1-\mathrm{Mn} 1-\mathrm{N} 1$ & $90.84(9)$ \\
\hline $\mathrm{O} 2-\mathrm{C} 11-\mathrm{C} 9$ & $114.0(3)$ & $\mathrm{O} 8-\mathrm{Mn} 1-\mathrm{N} 1$ & $176.23(10)$ \\
\hline $\mathrm{O} 2-\mathrm{C} 11-\mathrm{H} 11 \mathrm{~A}$ & 108.8 & $\mathrm{O} 4-\mathrm{Mn} 1-\mathrm{O} 6$ & $92.33(8)$ \\
\hline $\mathrm{C} 9-\mathrm{C} 11-\mathrm{H} 11 \mathrm{~A}$ & 108.8 & $\mathrm{O} 1-\mathrm{Mn} 1-\mathrm{O} 6$ & $89.24(9)$ \\
\hline $\mathrm{O} 2-\mathrm{C} 11-\mathrm{H} 11 \mathrm{~B}$ & 108.8 & $\mathrm{O} 8-\mathrm{Mn} 1-\mathrm{O} 6$ & $88.97(9)$ \\
\hline C9-C11-H11B & 108.8 & $\mathrm{~N} 1-\mathrm{Mn} 1-\mathrm{O} 6$ & $93.22(9)$ \\
\hline $\mathrm{H} 11 \mathrm{~A}-\mathrm{C} 11-\mathrm{H} 11 \mathrm{~B}$ & 107.6 & $\mathrm{O} 4-\mathrm{Mn} 1-\mathrm{O} 9$ & $88.58(9)$ \\
\hline $\mathrm{O} 4-\mathrm{C} 12-\mathrm{C} 9$ & $109.7(2)$ & $\mathrm{O} 1-\mathrm{Mn} 1-\mathrm{O} 9$ & $90.39(9)$ \\
\hline $\mathrm{O} 4-\mathrm{C} 12-\mathrm{H} 12 \mathrm{~A}$ & 109.7 & $\mathrm{O} 8-\mathrm{Mn} 1-\mathrm{O} 9$ & $86.27(9)$ \\
\hline $\mathrm{C} 9-\mathrm{C} 12-\mathrm{H} 12 \mathrm{~A}$ & 109.7 & $\mathrm{~N} 1-\mathrm{Mn} 1-\mathrm{O} 9$ & $91.54(10)$ \\
\hline $\mathrm{O} 4-\mathrm{C} 12-\mathrm{H} 12 \mathrm{~B}$ & 109.7 & O6-Mn1-O9 & $175.23(9)$ \\
\hline $\mathrm{C} 9-\mathrm{C} 12-\mathrm{H} 12 \mathrm{~B}$ & 109.7 & $\mathrm{O} 4-\mathrm{Mn} 2-\mathrm{O} 4$ & $180.000(1)$ \\
\hline $\mathrm{H} 12 \mathrm{~A}-\mathrm{C} 12-\mathrm{H} 12 \mathrm{~B}$ & 108.2 & $\mathrm{O} 4-\mathrm{Mn} 2-\mathrm{O}^{\mathrm{i}}$ & $89.03(8)$ \\
\hline $\mathrm{O} 5-\mathrm{C} 13-\mathrm{O} 6$ & $125.6(3)$ & $\mathrm{O} 4-\mathrm{Mn} 2-\mathrm{O}^{\mathrm{i}}$ & $90.98(8)$ \\
\hline $\mathrm{O} 5-\mathrm{C} 13-\mathrm{C} 14$ & $117.5(3)$ & $\mathrm{O} 4-\mathrm{Mn} 2-\mathrm{O} 7$ & $90.97(8)$ \\
\hline $\mathrm{O} 6-\mathrm{C} 13-\mathrm{C} 14$ & $117.0(3)$ & $\mathrm{O} 4-\mathrm{Mn} 2-\mathrm{O} 7$ & $89.02(8)$ \\
\hline $\mathrm{C} 13-\mathrm{C} 14-\mathrm{H} 14 \mathrm{~A}$ & 109.5 & $\mathrm{O} 7 \mathrm{i}-\mathrm{Mn} 2-\mathrm{O} 7$ & $180.000(1)$ \\
\hline $\mathrm{C} 13-\mathrm{C} 14-\mathrm{H} 14 \mathrm{~B}$ & 109.5 & $\mathrm{O} 4-\mathrm{i}-\mathrm{Mn} 2-\mathrm{O} 5$ & $88.78(8)$ \\
\hline
\end{tabular}


H14A-C14-H14B

C13-C14-H14C

$\mathrm{H} 14 \mathrm{~A}-\mathrm{C} 14-\mathrm{H} 14 \mathrm{C}$

H14B-C14-H14C

$\mathrm{O} 7-\mathrm{C} 15-\mathrm{O} 8$

$\mathrm{O} 7-\mathrm{C} 15-\mathrm{C} 16$

$\mathrm{O} 8-\mathrm{C} 15-\mathrm{C} 16$

C15-C16-H16A

C15-C16-H16B

$\mathrm{O} 1-\mathrm{C} 1-\mathrm{C} 2-\mathrm{C} 3$

$\mathrm{C} 6-\mathrm{C} 1-\mathrm{C} 2-\mathrm{C} 3$

$\mathrm{C} 1-\mathrm{C} 2-\mathrm{C} 3-\mathrm{C} 4$

$\mathrm{C} 2-\mathrm{C} 3-\mathrm{C} 4-\mathrm{C} 5$

$\mathrm{C} 3-\mathrm{C} 4-\mathrm{C} 5-\mathrm{C} 6$

$\mathrm{C} 4-\mathrm{C} 5-\mathrm{C} 6-\mathrm{C} 1$

$\mathrm{C} 4-\mathrm{C} 5-\mathrm{C} 6-\mathrm{C} 7$

$\mathrm{O} 1-\mathrm{C} 1-\mathrm{C} 6-\mathrm{C} 5$

$\mathrm{C} 2-\mathrm{C} 1-\mathrm{C} 6-\mathrm{C} 5$

$\mathrm{O} 1-\mathrm{C} 1-\mathrm{C} 6-\mathrm{C} 7$

$\mathrm{C} 2-\mathrm{C} 1-\mathrm{C} 6-\mathrm{C} 7$

$\mathrm{C} 5-\mathrm{C} 6-\mathrm{C} 7-\mathrm{N} 1$

$\mathrm{C} 1-\mathrm{C} 6-\mathrm{C} 7-\mathrm{N} 1$

$\mathrm{N} 1-\mathrm{C} 9-\mathrm{C} 10-\mathrm{O} 3$

$\mathrm{C} 12-\mathrm{C} 9-\mathrm{C} 10-\mathrm{O} 3$

$\mathrm{C} 11-\mathrm{C} 9-\mathrm{C} 10-\mathrm{O} 3$

$\mathrm{N} 1-\mathrm{C} 9-\mathrm{C} 11-\mathrm{O} 2$

$\mathrm{C} 12-\mathrm{C} 9-\mathrm{C} 11-\mathrm{O} 2$

$\mathrm{C} 10-\mathrm{C} 9-\mathrm{C} 11-\mathrm{O} 2$

$\mathrm{N} 1-\mathrm{C} 9-\mathrm{C} 12-\mathrm{O} 4$

$\mathrm{C} 10-\mathrm{C} 9-\mathrm{C} 12-\mathrm{O} 4$

$\mathrm{C} 11-\mathrm{C} 9-\mathrm{C} 12-\mathrm{O} 4$

C6- 7 - $-\mathrm{N} 1-\mathrm{C} 9$

$\mathrm{C} 6-\mathrm{C} 7-\mathrm{N} 1-\mathrm{Mn} 1$

C12-C9-N1-C7

$\mathrm{C} 10-\mathrm{C} 9-\mathrm{N} 1-\mathrm{C} 7$

$\mathrm{C} 11-\mathrm{C} 9-\mathrm{N} 1-\mathrm{C} 7$

$\mathrm{C} 12-\mathrm{C} 9-\mathrm{N} 1-\mathrm{Mn} 1$

$\mathrm{C} 10-\mathrm{C} 9-\mathrm{N} 1-\mathrm{Mn} 1$

$\mathrm{C} 11-\mathrm{C} 9-\mathrm{N} 1-\mathrm{Mn} 1$

$\mathrm{C} 2-\mathrm{C} 1-\mathrm{O} 1-\mathrm{Mn} 1$

$\mathrm{C} 6-\mathrm{C} 1-\mathrm{O} 1-\mathrm{Mn} 1$

$\mathrm{C} 9-\mathrm{C} 12-\mathrm{O} 4-\mathrm{Mn} 1$

$\mathrm{C} 9-\mathrm{C} 12-\mathrm{O} 4-\mathrm{Mn} 2$

$\mathrm{O} 6-\mathrm{C} 13-\mathrm{O} 5-\mathrm{Mn} 2$

$\mathrm{C} 14-\mathrm{C} 13-\mathrm{O} 5-\mathrm{Mn} 2$

$\mathrm{O} 5-\mathrm{C} 13-\mathrm{O} 6-\mathrm{Mn} 1$

$\mathrm{C} 14-\mathrm{C} 13-\mathrm{O} 6-\mathrm{Mn} 1$
109.5

109.5

109.5

109.5

124.7 (3)

$119.5(3)$

115.7 (3)

109.5

109.5

$178.6(3)$

$-1.2(5)$

$1.9(6)$

$-1.1(7)$

$-0.4(6)$

$1.0(5)$

$-175.3(4)$

$180.0(3)$

$-0.2(5)$

$-3.9(5)$

$175.9(3)$

$171.7(3)$

$-4.5(5)$

$51.4(4)$

$-65.0(3)$

$173.0(3)$

54.4 (4)

$167.1(3)$

$-70.3(4)$

42.2 (3)

164.4 (3)

$-73.1(3)$

$-177.5(3)$

1.9 (4)

$152.4(3)$

$32.1(4)$

$-91.0(3)$

$-27.1(3)$

$-147.4(2)$

89.5 (3)

$-164.9(2)$

$14.9(4)$

$-41.2(3)$

$122.8(2)$

$-9.9(6)$

169.9 (3)

$3.4(5)$

$-176.4(3)$
$\mathrm{O} 4-\mathrm{Mn} 2-\mathrm{O} 5$

$\mathrm{O} 7 \mathrm{i}-\mathrm{Mn} 2-\mathrm{O} 5$

$\mathrm{O} 7-\mathrm{Mn} 2-\mathrm{O} 5$

$\mathrm{O} 4-\mathrm{Mn} 2-\mathrm{O} 5^{\mathrm{i}}$

$\mathrm{O} 4-\mathrm{Mn} 2-\mathrm{O} 5^{\mathrm{i}}$

$\mathrm{O} 7^{\mathrm{i}}-\mathrm{Mn} 2-\mathrm{O} 5^{\mathrm{i}}$

$\mathrm{O} 7-\mathrm{Mn} 2-\mathrm{O} 5^{\mathrm{i}}$

$\mathrm{O} 5-\mathrm{Mn} 2-\mathrm{O} 5^{\mathrm{i}}$

$\mathrm{Mn} 2-\mathrm{O} 4-\mathrm{Mn} 1-\mathrm{O} 6$

$\mathrm{C} 12-\mathrm{O} 4-\mathrm{Mn} 1-\mathrm{O} 9$

$\mathrm{Mn} 2-\mathrm{O} 4-\mathrm{Mn} 1-\mathrm{O} 9$

$\mathrm{C} 1-\mathrm{O} 1-\mathrm{Mn} 1-\mathrm{O} 4$

$\mathrm{C} 1-\mathrm{O} 1-\mathrm{Mn} 1-\mathrm{O} 8$

$\mathrm{C} 1-\mathrm{O} 1-\mathrm{Mn} 1-\mathrm{N} 1$

$\mathrm{C} 1-\mathrm{O} 1-\mathrm{Mn} 1-\mathrm{O} 6$

$\mathrm{C} 1-\mathrm{O} 1-\mathrm{Mn} 1-\mathrm{O} 9$

C15-O8-Mn1-O4

$\mathrm{C} 15-\mathrm{O} 8-\mathrm{Mn} 1-\mathrm{O} 1$

$\mathrm{C} 15-\mathrm{O} 8-\mathrm{Mn} 1-\mathrm{N} 1$

$\mathrm{C} 15-\mathrm{O} 8-\mathrm{Mn} 1-\mathrm{O} 6$

$\mathrm{C} 15-\mathrm{O} 8-\mathrm{Mn} 1-\mathrm{O} 9$

C7-N1-Mn1-O4

C9-N1-Mn1-O4

C7-N1-Mn1-O1

C9-N1-Mn1-O1

C7-N1-Mn1-O8

C9-N1-Mn1-O8

C7-N1-Mn1-O6

C9-N1-Mn1-O6

C7-N1-Mn1-O9

C9-N1-Mn1-O9

$\mathrm{C} 13-\mathrm{O} 6-\mathrm{Mn} 1-\mathrm{O} 4$

C13-O6-Mn1-O1

$\mathrm{C} 13-\mathrm{O} 6-\mathrm{Mn} 1-\mathrm{O} 8$

C13-O6-Mn1-N1

$\mathrm{C} 13-\mathrm{O} 6-\mathrm{Mn1}-\mathrm{O} 9$

$\mathrm{C} 12-\mathrm{O} 4-\mathrm{Mn} 2-\mathrm{O} 4^{\mathrm{i}}$

$\mathrm{Mn} 1-\mathrm{O} 4-\mathrm{Mn} 2-\mathrm{O} 4^{\mathrm{i}}$

$\mathrm{C} 12-\mathrm{O} 4-\mathrm{Mn} 2-\mathrm{O}^{\mathrm{i}}$

$\mathrm{Mn} 1-\mathrm{O} 4-\mathrm{Mn} 2-\mathrm{O}^{\mathrm{i}}$

$\mathrm{C} 12-\mathrm{O} 4-\mathrm{Mn} 2-\mathrm{O} 7$

$\mathrm{Mn} 1-\mathrm{O} 4-\mathrm{Mn} 2-\mathrm{O} 7$

$\mathrm{C} 12-\mathrm{O} 4-\mathrm{Mn} 2-\mathrm{O} 5$

$\mathrm{Mn} 1-\mathrm{O} 4-\mathrm{Mn} 2-\mathrm{O} 5$

$\mathrm{C} 12-\mathrm{O} 4-\mathrm{Mn} 2-\mathrm{O}^{\mathrm{i}}$

$\mathrm{Mn} 1-\mathrm{O} 4-\mathrm{Mn} 2-\mathrm{O}^{\mathrm{i}}$
$91.22(8)$

$90.48(10)$

$89.52(10)$

$91.22(8)$

$88.78(8)$

$89.52(10)$

$90.48(10)$

$180.000(1)$

$-51.58(12)$

$-71.9(2)$

$123.73(12)$

$-2.9(10)$

$164.2(3)$

$-13.6(3)$

$-106.8(3)$

$78.0(3)$

$-15.7(3)$

$165.8(3)$

$-158.1(13)$

$76.5(3)$

$-103.6(3)$

-173.7 (3)

$5.8(2)$

$5.1(3)$

$-175.4(2)$

$-30.9(16)$

148.6 (14)

94.4 (3)

$-86.1(2)$

$-85.3(3)$

$94.2(2)$

26.9 (3)

$-159.5(3)$

-73.4 (3)

109.7 (3)

-74.0 (11)

$150(48)$

$-47(48)$

$-23.4(2)$

139.47 (13)

$156.6(2)$

-40.53 (13)

-113.9 (2)

48.97 (13)

66.1 (2)

$-131.03(13)$ 


$\begin{array}{llll}\mathrm{O} 8-\mathrm{C} 15-\mathrm{O} 7-\mathrm{Mn} 2 & -15.7(5) & \mathrm{C} 15-\mathrm{O} 7-\mathrm{Mn} 2-\mathrm{O} 4^{\mathrm{i}} & -148.5(3) \\ \mathrm{C} 16-\mathrm{C} 15-\mathrm{O} 7-\mathrm{Mn} 2 & 163.0(2) & \mathrm{C} 15-\mathrm{O} 7-\mathrm{Mn} 2-\mathrm{O} 4 & 31.5(3) \\ \mathrm{O} 7-\mathrm{C} 15-\mathrm{O} 8-\mathrm{Mn} 1 & 3.5(5) & \mathrm{C} 15-\mathrm{O} 7-\mathrm{Mn} 2-\mathrm{O} 7^{\mathrm{i}} & 112(100) \\ \mathrm{C} 16-\mathrm{C} 15-\mathrm{O} 8-\mathrm{Mn} 1 & -175.3(2) & \mathrm{C} 15-\mathrm{O} 7-\mathrm{Mn} 2-\mathrm{O} 5 & -59.7(3) \\ \mathrm{C} 12-\mathrm{O} 4-\mathrm{Mn} 1-\mathrm{O} 1 & 9.0(9) & \mathrm{C} 15-\mathrm{O} 7-\mathrm{Mn} 2-\mathrm{O} 5^{\mathrm{i}} & 120.3(3) \\ \mathrm{Mn} 2-\mathrm{O} 4-\mathrm{Mn} 1-\mathrm{O} 1 & -155.3(8) & \mathrm{C} 13-\mathrm{O} 5-\mathrm{Mn} 2-\mathrm{O} 4^{\mathrm{i}} & 166.7(3) \\ \mathrm{C} 12-\mathrm{O} 4-\mathrm{Mn} 1-\mathrm{O} 8 & -157.9(2) & \mathrm{C} 13-\mathrm{O} 5-\mathrm{Mn} 2-\mathrm{O} 4 & -13.3(3) \\ \mathrm{Mn} 2-\mathrm{O} 4-\mathrm{Mn} 1-\mathrm{O} 8 & 37.79(13) & \mathrm{C} 13-\mathrm{O} 5-\mathrm{Mn} 2-\mathrm{O} 7^{\mathrm{i}} & -104.3(3) \\ \mathrm{C} 12-\mathrm{O} 4-\mathrm{Mn} 1-\mathrm{N} 1 & 19.8(2) & \mathrm{C} 13-\mathrm{O} 5-\mathrm{Mn} 2-\mathrm{O} 7 & 75.7(3) \\ \mathrm{Mn} 2-\mathrm{O} 4-\mathrm{Mn} 1-\mathrm{N} 1 & -144.53(13) & \mathrm{C} 13-\mathrm{O} 5-\mathrm{Mn} 2-\mathrm{O} 5^{\mathrm{i}} & 30(100) \\ \mathrm{C} 12-\mathrm{O} 4-\mathrm{Mn} 1-\mathrm{O} 6 & 112.8(2) & & \end{array}$

Symmetry code: (i) $-x+1,-y+2,-z+2$.

Hydrogen-bond geometry $\left(\AA,{ }^{\circ}\right)$

\begin{tabular}{|c|c|c|c|c|}
\hline$D-\mathrm{H} \cdots A$ & $D-\mathrm{H}$ & $\mathrm{H} \cdots A$ & $D^{\cdots} A$ & $D-\mathrm{H} \cdots A$ \\
\hline $\mathrm{O} 9-\mathrm{H} 9 A \cdots \mathrm{O} 10^{\mathrm{ii}}$ & $0.85(1)$ & $1.97(1)$ & $2.806(4)$ & $167(3)$ \\
\hline $\mathrm{O} 9-\mathrm{H} 9 B \cdots \mathrm{O} 8^{\mathrm{iii}}$ & $0.85(1)$ & $2.23(3)$ & $3.008(3)$ & $153(5)$ \\
\hline $\mathrm{O} 9-\mathrm{H} 9 B \cdots \mathrm{O} 1^{\mathrm{iii}}$ & $0.85(1)$ & $2.61(3)$ & $3.322(3)$ & $142(5)$ \\
\hline $\mathrm{O} 10-\mathrm{H} 10 C \cdots \mathrm{O} 5^{\mathrm{iv}}$ & $0.85(1)$ & $2.06(1)$ & $2.907(4)$ & $176(5)$ \\
\hline $\mathrm{O} 10-\mathrm{H} 10 D^{\cdots} \cdots \mathrm{N} 2^{v}$ & $0.85(1)$ & $2.07(1)$ & $2.914(6)$ & $174(6)$ \\
\hline $\mathrm{O} 2-\mathrm{H} 2 \cdots \mathrm{O}^{\mathrm{vi}}$ & 0.82 & 2.55 & $3.362(5)$ & 172 \\
\hline $\mathrm{O} 3-\mathrm{H} 3 \cdots \mathrm{O}^{\mathrm{vii}}$ & 0.82 & 2.00 & $2.777(3)$ & 159 \\
\hline
\end{tabular}

Symmetry codes: (ii) $x, y+1, z$; (iii) $-x,-y+2,-z+2$; (iv) $-x+1,-y+1,-z+2$; (v) $x,-y+3 / 2, z+1 / 2$; (vi) $-x, y-1 / 2,-z+3 / 2$; (vii) $-x, y+1 / 2,-z+3 / 2$. 